GANIT J. Bangladesh Math. Soc. (ISSN 1606-3694) 29 (2009) 35-41

\title{
A STANDARD MODULO PRIESTLEY TOPOLOGICAL QUASI-VARIETY
}

\author{
S. N. Begum and M. R. Rahman \\ Department of Mathematics, ShahJalal University of Science and Technology \\ Sylhet 3114, Bangladesh \\ Email: snaher@yahoo.com
}

Received 26.11.06 Accepted 19.04.08

\begin{abstract}
We construct a topological quasi-variety which is not standard but standard modulo Priestley.
\end{abstract}

Key words: Standard topological quasi-variety, Boolean model, standard modulo Priestley.

\section{Introduction}

A structure $\tilde{M}=<M ; G, H, R, T>$ is said to be a topological structure if $\langle M ; T>$ is a topological space, $G$ is a set of finitary total operations, $H$ is a set of finitary partial operations and $R$ is a set of finitary relations on $M$. If the topological space $\langle M ; T\rangle$ is compact, then we say that $\tilde{M}$ is compact topological structure. A topological quasivariety generated by a finite topological structure $\tilde{M}=<M ; G, H, R, T>$ with discrete topology $T$ is the class $I S_{c} P^{+}(\tilde{M})$ of isomorphic copies of closed substructure of nonempty direct power of $\tilde{M}$. For convenience of notation we will write $Q_{T}(\tilde{M})$ for $I S_{c} P^{+}(\tilde{M})$.

\subsection{Definition of standardness}

Let $\tilde{M}$ be a topological structure. We say that a topological structure $\widetilde{X}=<X ; G^{X}, H^{X}, R^{X}, T^{X}>$ is the same type as $\tilde{M}$ if

(i) $<X ; T^{X}>$ is a topological space,

(ii) for each $g \in G \cup H \cup R$, there is a corresponding $g^{X} \in G^{X} \cup H^{X} \cup R^{X}$ of the same arity as that of $g$.

A topological structure $\tilde{X}=<X ; G^{X}, H^{X}, R^{X}, T^{X}>$ is said to be a Boolean structure (or Boolean model) of type $\langle G, H, R\rangle$ if

(i) $<X ; T^{X}>$ is a Boolean space (that is, compact totally disconnected space),

(ii) for each $n$-ary $h \in G \cup H$, the set $\operatorname{dom}\left(h^{X}\right)$ is a closed subset of $X^{n}$ and $h^{X}$ : $\operatorname{dom}\left(h^{X}\right) \rightarrow X$ is continuous, and 
(iii) if $r \in R$ is $n$-ary, then $r^{X}$ is a closed subset of $X^{n}$.

We will omit the superscripts on $G^{X}, H^{X}, R^{X}, T^{X}$ where there is no danger of ambiguity.

An atomic formula of type $\langle G, H, R>$ is an expression of either of the forms

$$
t_{1} \approx t_{2} \text { or } r\left(t_{1}, t_{2}, \cdots, t_{n}\right)
$$

where $t_{1}, t_{2}, \cdots, t_{n}$ are terms built from the function symbols in $G \cup H$ and $r \in R$ is an $n$ ary relation symbol. A quasi-atomic formula of type $\langle G, H, R\rangle$ is an expression of one of the forms

$$
\alpha \text { or } \underset{i \in I}{\vee} \neg \beta_{i} \text { or } \underset{i \in I}{\wedge} \beta_{i} \Rightarrow \alpha
$$

where $\alpha$ and each $\beta_{i}$ are atomic formulæ and $I$ is a finite set.

Let $\Sigma$ be a set of quasi-atomic formulæ. We denote by $\operatorname{Mod}_{T}(\Sigma)$ the class of all Boolean structures which satisfy each quasi-atomic formula in $\Sigma$. The collection of all quasiatomic formulæ that hold in $\tilde{M}$ forms the quasi-atomic theory of $\tilde{M}$ which is denoted by $T h_{q a}(\tilde{M})$.

We say that $Q_{T}(\tilde{M})$ is a standard topological quasi-variety, or that $\tilde{M}$ is standard, if $Q_{T}(\tilde{M})$ is exactly the class of all Boolean models of the quasi-atomic theory of $\tilde{M}$, in symbols,

$$
Q_{T}(\tilde{M})=\operatorname{Mod}_{T}\left(\operatorname{Th}_{q a}(\tilde{M})\right)
$$

We say that a subset $\Sigma \subseteq T h_{q a}(\tilde{M})$ axiomatizes $Q_{T}(\tilde{M})$ provided that $Q_{T}(\tilde{M})=\operatorname{Mod}_{T}(\Sigma)$. If $Q_{T}(\tilde{M})$ is axiomatizable, then it is certainly standard, and the axioms provide a description of its members.

Most of the cases $Q_{T}(\tilde{M})$ are not standard, but we can often describe them by assuming that the underlying ordered set is a Priestley space (that is, compact totally orderdisconnected space). This is the reason that we are interested in studying standardness modulo Priestley.

\subsection{Definition of Standard modulo Priestley}

Let $\tilde{M}=<M ; G, H, \leq, T>$ be a finite topological ordered partial algebra. A topological structure $\tilde{X}=<X ; G^{X}, H^{X}, \leq^{X}, T^{X}>$ of the same type $<G, H, \leq>$ as $\tilde{M}$ is said to be a Priestley structure (or Priestley model) of type $\langle G, H\rangle$ if

(i) $\tilde{X}=<X ; \leq^{X}, T^{X}>$ is a Priestley space,

(ii) If $g \in G \cup H$ is an $n$-ary operation, then $\operatorname{dom}\left(g^{X}\right)$ is a closed subset of $X^{n}$ and $g^{X}$ : $\operatorname{dom}\left(g^{X}\right) \rightarrow X$ is continuous. 
Let $\Sigma$ be a set of quasi-atomic formulæ satisfied by $\tilde{M}$. We denote the class of all Priestley models which satisfy each quasi-atomic formula in $\Sigma$ by $\operatorname{Mod}_{P}(\Sigma)$. The class of all Priestley models of the quasi-atomic theory of $\tilde{M}$ is denoted by $\operatorname{Mod}_{P}\left(\operatorname{Th}_{q a}(\tilde{M})\right)$. We say that $Q_{T}(\tilde{M})$ is standard modulo Priestley, or that $\tilde{M}$ is standard modulo Priestley, if $Q_{T}(\tilde{M})$ is exactly the class of all Priestley models of the quasi-atomic theory of $\tilde{M}$. In symbols,

$$
Q_{T}(\tilde{M})=\operatorname{Mod}_{P}\left(\operatorname{Th}_{q a}(\tilde{M})\right)
$$

Throughout this paper, if $\langle X ; T\rangle$ is a finite topological space, then it will be assumed that $T$ is the discrete topology.

A natural question is: Which finite topological ordered partial algebras $\tilde{M}$ generate a topological quasi-variety that is standard modulo Priestley? By the definition, the category of the Priestley space is standard modulo Priestley. By [6, Theorem 4.2], every finite Boolean unar is standard. Every finite anti-chain is term equivalent to a finite Boolean unar and hence every finite anti-chain is standard. Begum [3] proved that all two element topological ordered unars are standard modulo Priestley. She also proved that all three and four-element topological chain with an order-preserving operation is standard modulo Priestley.

In Section 2, we give a detail background which we need in this paper. In Section 3, we consider a quasi-variety generated by a four element Boolean ordered unar which is neither a chain nor an anti-chain. We show that the quasi-variety is not standard but standard modulo Priestley.

\section{Preliminaries}

The notion of standardness was first introduced in [6]. The standardness problem arises from the question: Which structures are in and what do they look like? The Preservation Theorem gives us a set of axioms which satisfies each member of $I S_{c} P^{+}(\tilde{M})$.

Theorem 2.1 (The Preservation Theorem 1.4.3 [5]) Let $\tilde{M}$ be a finite topological structure and let $X \in I S_{c} P^{+}(\tilde{M})$. Then $\boldsymbol{X}$ is a Boolean structure which satisfies every quasi-atomic formula that is satisfied by $\tilde{M}$.

The following Lemma is compiled from [7, Lemma 11.2 and Exercise 11.14].

Lemma 2.2 Let $\tilde{X}=<X ; \leq, T>$ be a Priestley space.

(i) let $Y$ be a close downset in $X$ and let $x \notin Y$. Then there exists a clopen downset $U$ such that $Y \subseteq U$ and $x \notin U$.

(ii) ${ }^{\downarrow} y$ and ${ }^{\uparrow} y$ are closed for each $y \in X$.

(iii) If $Y \subseteq X$ is closed in $X$, then ${ }^{\downarrow} Y$ and ${ }^{\uparrow} Y$ are closed in $X$. 
To prove a topological structure $\tilde{M}=<M ; g, \leq, T>$ is non-standard, we will often use the following lemma which is due to [3].

Lemma 2.3 Let $\tilde{M}=<M ; G, \leq, T>$ be a finite topological ordered unary algebra and let $\widetilde{X}=<X ; G, \leq, T>$. Then the following are equivalent:

(i) $\tilde{X} \in Q_{T}(\tilde{M})$,

(ii) $\tilde{X}$ is a Boolean ordered unary algebra (that is, $\langle X ; T\rangle$ is a Boolean space and for all $g \in G$ the map $g: X \rightarrow X$ is a continuous and $\leq i$ a closed order relation) such that

(Sep) for all $x, y \in X$ with $x \not y$, there exist a continuous g-preserving and $\leq-$ preserving map $\alpha: X \rightarrow M$ with $\alpha(x) \not \alpha(y)$.

(iii) each $g \in G$ is a unary map on $X$, the binary relation $\leq$ on $X$ is an anti-symmetric and (Sep) holds.

Note that this lemma shows that provided we can establish (Sep) then we do not need to prove that the maps $g: X \rightarrow X$ are continuous! We may now use these tools to show that specific Boolean ordered unars are non-standard.

Let $\tilde{M}=<M ; g, \leq, T>$ be a finite topological ordered unar. Then we say that $\tilde{M}$ is nonstandard via Priestley if there exists a Boolean structure $\widetilde{X}=<X ; g, \leq, T>$ such that

(a) $\tilde{X}$ is locally finite,

(b) every finite substructure of $\tilde{X}$ is in $Q_{T}(\tilde{M})$, and

(c) $<X ; \leq, T>$ is not a Priestley space.

Since every underlying ordered space of every member of $Q_{T}(\tilde{M})$ is a Priestley space, it follows that non-standard via Priestley implies non-standard. Indeed, one of the standard ways to prove that a finite topological ordered unar is non-standard is to prove that it is non-standard via Priestley.

Proving that $\tilde{M}$ is non-standard via Priestley has the added advantage that it is an inherent property, that is, it goes up to larger quasi-varieties.

Lemma 2.4 ([1]) Let $\tilde{M}=<M ; g, \leq, T>$ and $\tilde{N}=<N ; g, \leq, T>$ be finite topological ordered unars. If $\tilde{M}$ is non-standard via Priestley and $\tilde{M} \in Q_{T}(\tilde{N})$, then $\tilde{N}$ is also nonstandard via Priestley.

Proof. Let $\tilde{X}$ be an example that shows that $\tilde{M}$ is non-standard via Priestley. Since $\tilde{M} \in Q_{T}(\tilde{N})$ implies that $Q_{T}(\tilde{M}) \subseteq Q_{T}(\widetilde{N})$ it follows immediately that $\widetilde{X}$ also shows that $\widetilde{N}$ is non-standard via Priestley. 
Corollary 2.5 ([1]). Let $\tilde{M}$ and $\widetilde{N}$ be finite topological ordered unars. If $\tilde{M}$ is nonstandard via Priestley and $\widetilde{M}$ is a substructure of $\widetilde{N}$, then $\widetilde{N}$ is also non-standard via Priestley.

Lemma 2.6 Let $\tilde{M}=<M ; g, \leq, T>$ be a finite topological ordered unary algebra. Let $\Sigma$ be a set of axioms satisfied by $\tilde{M}$. If $\tilde{X} \in Q_{T}(\tilde{M})$, then $\tilde{X} \in \operatorname{Mod}_{T}(\Sigma)$ and $<X ; \leq, T>$ is a Priestley space.

Proof. Let $\tilde{X} \in Q_{T}(\tilde{M})$. Then by the Preservation Theorem 2.1, $\widetilde{X} \in \operatorname{Mod}_{T}(\Sigma)$. To prove $<X ; \leq, T>$ is a Priestley space, let $x, y \in X$ with $x \not \leq y$. Since $\tilde{X} \in Q_{T}(\tilde{M})$, by the Lemma 2.3, there exists a morphism $\alpha: \tilde{X} \rightarrow \tilde{M}$ such that $\alpha(x) \leq \alpha(y)$. The clopen downset $\alpha^{-1}\left({ }^{\downarrow} \alpha(y)\right)$ in $X$ contains $y$ but not $x$. Hence $\langle X ; \leq, T>$ is a Priestley space.

Because of the above Lemma we are guaranteed, for topological unary algebras, that if $\tilde{X} \in Q_{T}(\tilde{M})$, then $\tilde{X} \in \operatorname{Mod}_{P}\left(\operatorname{Th}_{q a}(\tilde{M})\right)$ so that to show $Q_{T}(\tilde{M})=\operatorname{Mod}_{P}\left(\operatorname{Th}_{q a}(\tilde{M})\right)$ we need only

- write down a set $\Sigma$ of axioms that is satisfied by $\tilde{M}$, and

- show that if $\tilde{X}=<X ; G, \leq, T>$ is a Boolean model of $\Sigma$ such that $\langle X ; \leq, T>$ is Priestley space, then condition (Sep) of Lemma 2.3 holds.

This is the "standard method" to show that a finite topological ordered unary algebra $\widetilde{M}=<M ; G, \leq, T>$ is standard modulo Priestley.

\section{A non-standard structure which are Standard modulo priestley}

In this section we consider the topological quasi-varieties generated by a four-element Boolean ordered unar $M_{4}$ (see Figure 2) which is neither a chain nor an anti-chain. We show that it is non-standard but standard modulo Priestley.

If we consider the structure $M_{2}$ given in Figure 1 where line indicate the relation and arrow line indicate the unary operation, then by $[1,2]$, we have $M_{2}$ is non-standard.

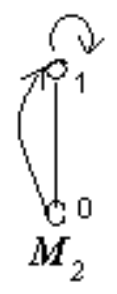

Figure-1

Now we construct our main topological quasi-variety. Consider the topological structure $\widetilde{M}_{4}=<\{0, a, b, 1\} ; g, \leq, T>$ given in the following Figure 2. 


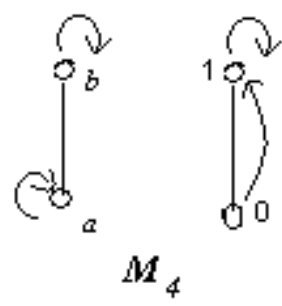

Figure 2: Four-element topological ordered unars

Observe that each $\tilde{X} \in Q_{T}\left(\tilde{M}_{4}\right)$ satisfies the following set $\Sigma_{M_{4}}$ of axioms:

(i) $\leq$ is an order relation (i.e., reflexive, symmetry and transitive),

(ii) $x \leq y \Rightarrow g(x) \leq g(y)$

(iii) $g(x)=g^{2}(x)$

(iv) $g(x) \leq g(y)$ and $g(y)=y \Rightarrow x \leq y$

(v) $g(x)=x$ and $x \leq y \Rightarrow g(y)=y$

Theorem $3.1 M_{4}$ is non-standard.

Proof. Observe that $M_{2}$ is a substructure of $M_{4}$. By [1] $M_{2}$ is non-standard via Priestley. Then by Corollary 2.5, $M_{4}$ is non-standard via Priestley and hence nonstandard.

Theorem 3.2 $Q_{T}\left(\tilde{M}_{4}\right)=\operatorname{Mod}_{P}\left(\Sigma_{M_{4}}\right)$ and hence $Q_{T}\left(\tilde{M}_{4}\right)$ is standard modulo Priestley.

Proof. Let $\widetilde{X} \in \operatorname{Mod}_{T}\left(\Sigma_{M_{4}}\right)$ and $\langle X ; \leq, T>$ be a Priestley space. In order to apply Lemma 2.3, let $x, y \in X$ with $x \leq y$.

Case-1 $g(x) \not g(y)$. Since $<X ; \leq, T>$ is a Priestley space, there exists a clopen upset $U$ containing $g(x)$ but not $g(y)$. Thus $g^{-1}(U)$ contains $x$ but not $y$. Define a map $\alpha: X \rightarrow M$ by

$$
\alpha(z)=\left\{\begin{array}{l}
b \text { if } z \in g^{-1}(U) \\
a \text { if } z \in X \backslash g^{-1}(U) .
\end{array}\right.
$$

Since $U$ is an upset, by (i), we have $g^{-1}(U)$ is an upset and hence $X \backslash g^{-1}(U)$ is a downset. Therefore, $\alpha$ preserves $\leq$. By (ii), we have $g\left(g^{-1}(U)\right) \subseteq g^{-1}(U)$ and $g\left(X \backslash g^{-1}(U)\right) \subseteq X \backslash g^{-1}(U)$. Hence $\alpha$ preserves $g$. Moreover, $\alpha(x)=b \leq a=\alpha(y)$ and therefore $\alpha$ is the required separating morphism.

Case-2 $g(x) \leq g(y)$. By (iv), we have $g(y) \neq y$. Assume $g(x)=x$. Let $U:=f i x(g)$ be the set of fixed points of $X$. Since $g$ is continuous, $U$ is closed. Using (v) we can prove that $U$ 
is an upset. Hence, by Lemma 2.2, there is a clopen upset $V$ containing $U$ but not $y$. Define a map $\alpha: X \rightarrow M$ by

$$
\alpha(z)= \begin{cases}1 & \text { if } z \in V \\ 0 & \text { if } z \in X \backslash V .\end{cases}
$$

Here $V$ is an upset and $X \backslash V$ is a down set whence $g$ preserves $\leq$. Now for all $u \in X$ we have, by (ii), $g(u) \in U$ and hence $g(u) \in V$. Therefore, $g(V) \subseteq V$ and $g(X \backslash V) \subseteq V$. Thus, $\alpha$ preserves $g$. Moreover $\alpha(x)=1 \nless \alpha(y)=0$ and so $\alpha$ is the required separating morphism.

Again assume $g(x) \neq x$. and again let $U:=f i x(g)$. As above, $U$ is a closed upset. Define $V=U \cup^{\uparrow} x$ By Lemma 2.2, we have ${ }^{\uparrow} x$ is closed. Hence $V$ is a closed upset containing $x$ but not $y$. Hence, by Lemma 2.2, there exists a clopen upset $W$ containing $V$ but not $y$.

Define a map $\alpha: X \rightarrow M$ by

$$
\alpha(z)= \begin{cases}1 & \text { if } z \in W \\ 0 & \text { if } z \in X \backslash W .\end{cases}
$$

Since for all $u \in X$, we have $g(u) \in U \subseteq V \subseteq W$. Therefore, $\alpha$ is the required separating morphism of $x$ and $y$ with $\alpha(x)=1 \leq \alpha(y)=0$. Hence $M_{4}$ is standard modulo Priestley.

\section{REFERENCES}

1. S.N. Begum, A Study of the Standardness Problem for Ordered Unary Algebras, Masters Thesis, La Trobe University, Australia, (2003).

2. S.N. Begum, Standardness for two element Boolean ordered unars, SUST Studies, 6, no. 1 (2005), 77-83.

3. S.N. Begum, Standard Modulo Priestley for two-element topological ordered unar, SUST Studies, 7, no. 1, 43-51, (2007).

4. S.N. Begum, D.M. Clark, B.A. Davey and N. Perkal, Standardness for ordered unary algebras, in preparation.

5. D.M. Clark and B.A. Davey, Natural Dualities for the Working Algebraist, Cambridge University Press, Cambridge, (1998).

6. D.M. Clark, B.A. Davey, M. Haviar, J.G. Pitkethley and M.R. Talukder, Stan-dard topological quasivarieties, Houstan Journal of Mathematics, 29, 859-887, (2003).

7. B.A. Davey and H.A. Priestley, Introduction to Lattices and Order, second edition, Cambridge University Press, Cambridge, (2002).

8. H.A. Priestley, Representation of distributive lattices by means of ordered Stone spaces, Bull. London Math. Soc. 2, 186-190, (1970).

9. H.A. Priestley, Ordered topological spaces and the representation of distributive lattices, Proc. London Math. Soc. (3) 24, 507-530, (1972).

10. Stralka, A partially ordered space which is not a Priestley space, Semigroup Forum 20, 293-297, (1980). 